\title{
Un método para aprendizaje de problemas elusivos de Resistencia de Materiales mediante técnicas web A web-based tool for learning of some cumbersome problems on Strength of Materials
}

\author{
Juan Carlos Mosquera ${ }^{1}$, Isabel Chiyón², Luis Cueto-Felgueroso ${ }^{3}$, Iván Muñoz ${ }^{1}$ \\ juancarlos.mosquera@upm.es, isabel.chiyon@udep.pe, luis.cueto@upm.es, ivan.munoz@upm.es \\ ${ }^{1}$ Departamento de Mecánica de Medios \\ Continuos y Teoría de estructuras \\ Universidad Politécnica de Madrid \\ Madrid, España \\ ${ }^{3}$ Departamento de Ingeniería Civil: \\ Hidráulica, Energía y Medio Ambiente \\ Universidad Politécnica de Madrid \\ Madrid, España
}

\begin{abstract}
Resumen- En la docencia de ciertas asignaturas troncales de ingeniería se han identificado conceptos fundamentales, ligados al razonamiento espacial, cuyas aplicaciones prácticas inmediatas resultan a menudo elusivas a los alumnos. Son conceptos esenciales que se aplican posteriormente en materias avanzadas de cursos más altos del Grado o Master. Nociones tales como equilibrio, estabilidad, continuidad o esfuerzos son entendidas a priori por los alumnos. Pero para algunos, su aplicación a casos canónicos que requieren una cierta visión espacial se vuelve en ocasiones compleja y difícil de dominar. En este trabajo se muestra un método interactivo basado en técnicas web sencillas (HTML, JavaScript, librerías JQuery y minivídeos), orientado a que los estudiantes de Resistencia de Materiales puedan entender los conceptos de distribuciones de esfuerzos a lo largo de la estructura, vislumbrar sus zonas más solicitadas y analizar cómo influye su configuración geométrica (sus dimensiones y la sustentación) y de flexibilidad (sus propiedades materiales y mecánicas) sobre su respuesta ante ciertas acciones. Esta técnica es igualmente aplicable en Hidráulica (flujo en canales o tuberías). Así, los alumnos pueden aprender a su propio ritmo. Al mismo tiempo, esta herramienta permite llevar a cabo las técnicas de evaluación por pares y aula invertida.
\end{abstract}

Palabras clave: aula invertida, resistencia de materiales, razonamiento espacial, aprendizaje experiencial.

Abstract- Some fundamental branches are considered among the most difficult introductory courses in undergraduate Engineering education over the years. A number of paramount concepts are often hard to be understood by the Civil Engineering students. They should master various basic issues for further applications to advanced disciplines on practical structural design and projects. In this work a supplementary tool for learning support consisting of an interactive web-based learning assistant for fundamentals of Strength of Materials is presented. It is written in HTML5, JavaScript and JQuery frameworks, includes short videos and aims to ease the students' learning process based on a problem-solving strategy. The tool encompasses a repository of interactive exercises and problems in structural analysis. On the one hand, a group of students is prompted to use these materials whereas the rest of them follow the classical classroom sessions. Their achievements are compared with each other. On the other hand, this tool is used as an aid for peerassessment among students and for flipped classroom projects. The purposes are to enhance students' learning and increase the figures of passing students. Keywords: flipped classroom, Strength of Materials, spatial
reasoning, experiential learning.

\section{INTRODUCCIÓN}

Existe actualmente una gran variedad de estudios sobre los factores que influyen sobre los logros del aprendizaje, que abarcan desde los parámetros físicos, tales como el diseño de aulas, iluminación, calidad del aire y temperatura (Barrett, P., Davies, Zhang y Barrett, L., 2015), a los instrumentos o técnicas que inciden sobre niveles avanzados de la pirámide de aprendizaje (Lord, 2007). Con la implantación del EEES se ha potenciado el papel de las TICs para generar nuevos recursos y métodos (Gallego, Gámiz y Gutiérrez, 2010), que ofrecen oportunidades para orientar estrategias de aprendizaje de los alumnos basadas en competencias. En este sentido, las webs educativas constituyen una vía eficaz para alcanzar resultados del aprendizaje tales como comprobar, aplicar, poner en práctica, analizar e incluso evaluar (Maquilón, García-Sánchez. y Mirete, 2014). Por una parte, facilitan a los alumnos aprender a su propio ritmo a través de dispositivos electrónicos que suelen manejar a diario y con gran destreza (El-Sawy y Sweedan, 2010; Mirete, García y Sánchez, 2011). Por otra, contribuyen a la colaboración entre universidades en tanto que tarea de internacionalización (Ministerio de Educación, Cultura y Deporte, 2014; Unesco, 2015).

Este trabajo forma parte de una colaboración entre la Universidad Politécnica de Madrid (España) y la Universidad de Piura (Perú). Versa sobre asignaturas de los cursos básicos de ingeniería, tradicionalmente consideradas difíciles por los alumnos, quienes están acostumbrados inicialmente a aprender a base de memoria funcional (Marcé-Nogué, Gil, Pérez y Sánchez, 2013). El método propuesto, basado en recursos web asequibles para ser implementados en cualquier unidad docente, permite al alumnado ejercitarse en los niveles avanzados de la pirámide de aprendizaje, consolidando a la vez capacidades como el razonamiento espacial, la evaluación 
y el análisis de la respuesta de un sistema a los efectos de determinadas acciones conocidas.

La herramienta presentada pertenece al ámbito de una web didáctica. Por la forma en que está concebida e implementada (código abierto HTML5, JavaScript y JQuery), se puede aplicar en asignaturas que conllevan el aprendizaje autónomo basado en problemas, como son la Resistencia de Materiales o la Hidráulica. Asimismo, se puede aplicar a otras materias.

Con esta metodología se persigue que los alumnos puedan aprender experimentando virtualmente en su ordenador personal sobre modelos ingenieriles sencillos. Ellos pueden elegir los datos (geometría, materiales, secciones, valores y posición de las acciones y de las condiciones de contorno) y visualizar en tiempo real cuál es la respuesta del sistema. En el caso de una viga, por ejemplo, está expresada en términos de esfuerzos y movimientos. De esta manera, los alumnos pueden llegar a intuir o vislumbrar las zonas más solicitadas de una estructura ante unas acciones determinadas. Este aprendizaje les servirá para poder fijar los conceptos teóricos que fundamentan el comportamiento estructural y que históricamente hemos detectado que les cuesta mucho adquirir: deformaciones, flexibilidad y rigidez, inercia a flexión o sustentación, entre otros.

Por una parte, este instrumento de aprendizaje experiencial es a la vez para el alumnado un simulador virtual de lo que visualizan durante las prácticas experimentales de laboratorio. Es decir, una vez que el alumno ha participado o asistido a un ensayo de laboratorio durante el curso académico, puede reproducir virtualmente dicho ensayo en su ordenador, a través de esta herramienta web de simulación numérica (MosqueraFeijoo, Plaza-Beltrán \& González-Rodrigo, 2015). Por ejemplo, se puede medir en una práctica de laboratorio el máximo descenso en el centro de una viga cuando una carga se desplaza a lo largo de la misma. Dado que se dispone de una aplicación web que versa sobre líneas de influencia en vigas, el alumno puede replicar en su casa lo visualizado en el laboratorio. De este modo el alumno puede aprender a su ritmo y en su casa, a partir de lo presenciado en el ensayo. Ello permitirá dedicar mayor número de clases a la realización de proyectos y resolución de problemas.

Por otra parte, la herramienta web posibilita que los alumnos puedan configurar prácticas bajo la modalidad de "aula invertida" para ser transmitidas en presentaciones a los demás compañeros en el aula. Su alcance es posibilitar la visualización y comprensión de en qué consisten las características estructurales, qué es la respuesta del sistema y cómo se debe instrumentar un sistema en un caso real para poder medir eficazmente aquellas magnitudes que permitan identificar sus características y su respuesta.

\section{CONTEXTO}

El público objetivo son los estudiantes de asignaturas tecnológicas fundamentales de ingeniería; este equipo de trabajo se centra en Resistencia de Materiales y en Hidráulica.

Actualmente existe una gran diversidad de herramientas de autoaprendizaje en el ámbito de las ingenierías relacionadas con dichas materias. Hay diversidad de buscadores en Internet que facilitan su localización con criterios de búsqueda del tipo “apps gratuitas para estudiantes de ingeniería”. Por lo general, dichas apps usualmente calculan la respuesta de una estructura a partir de unos datos introducidos por el usuario. Se ha constatado que un cierto número de alumnos las utilizan por su cuenta en sus tabletas o teléfonos móviles.

La aspiración del proyecto actual presenta una singularidad: no son simples programas, páginas web o "apps" que concluyen en una solución a modo de caja negra con ayudas puntuales al usuario, sino que plantean una interacción mayor con el mismo, de forma que puede simular o experimentar virtualmente en tiempo real la variación en la respuesta estructural cuando varían las condiciones de las acciones, de sustentación o las características propias del sistema. En este documento se presenta un ejemplo de realización.

En el caso de la asignatura de Hidráulica, se prevé implementar esta metodología para el estudio de flujo en un tramo de canal o tubería cuando varían las condiciones geométricas o piezométricas en los contornos, pendientes, secciones, obstáculos, etc.

\section{A. Objetivos}

El entorno que se presenta en este documento consiste en un portal web de contenidos de la asignatura de Resistencia de Materiales, cuyos objetivos principales son:

- Incorporar mejoras en los procesos de aprendizaje de los alumnos de las asignaturas antes indicadas. Están concebidas para promover el aprendizaje experiencial, especialmente de aquellos estudiantes que tienen mayor dificultad de adquirir las competencias específicas, para que puedan superar las diferencias de nivel.

- Desarrollar una colección de aplicaciones interactivas, en español y en inglés, accesibles vía Web a la comunidad universitaria, para el aprendizaje de Resistencia de Materiales e Hidráulica.

- Incorporar mejoras específicas en la práctica docente de las asignaturas: páginas web con una colección de problemas que aglutinan los conocimientos y capacidades que los alumnos deben adquirir y dominar, así como para simular prácticas o ensayos de laboratorio.

\section{B. Contribución a la mejora de la calidad}

La experiencia indica que existe gran disparidad en la consecución de competencias entre los estudiantes de dichas asignaturas tecnológicas fundamentales. Se admite que el uso de las TICs puede ayudarles en este proceso: el aprendizaje experiencial del alumno puede verse facilitado con la inclusión de ciertas herramientas de simulación, utilizables en sus ordenadores y que incluyen breves accesorios multimedia (mini-vídeos o marcadores de enlace a realidad aumentada) para ayudarles con los conceptos, fundamentos y desarrollos que les suelen resultar elusivos año tras año. Pueden aprender a su propio ritmo y además elegir el idioma (español o inglés). Consideramos además que estas herramientas pueden cubrir además una deficiencia en los materiales y recursos disponibles para los alumnos.

\section{DESCRIPCIÓN}

\section{A. Características}

El recurso consiste en un portal web que contiene un catálogo de problemas interactivos de la asignatura, en los que se manejan los conceptos fundamentales cuya aplicación 
avanzada les cuesta asimilar. Cada alumno puede estudiar a su conveniencia cualesquiera problemas de la colección; en cada problema, puede configurar los datos. Las páginas web están programadas para devolver al instante los resultados correspondientes. Las características básicas de este recurso disponible en la web son:

- El texto de las prácticas y de las herramientas de simulación se realiza en español y en inglés, para dotarlas de mayor alcance internacional.

- Constituyen material docente para asignaturas impartidas en títulos de Grado en la Universidad Politécnica de Madrid y en la Universidad de Piura (Perú), la cual desde hace un año se ha implicado en la aplicación de TICs a la innovación educativa y ha formado un grupo de trabajo.

- Incluyen material de apoyo multimedia con varios minivídeos de acompañamiento de corta duración en cada práctica (menos de 90 segundos), con objeto de que un alumno pueda, adaptativamente, guiar o fortalecer su aprendizaje. Estas inclusiones también dejan una puerta a que sin dificultad se puedan ubicar marcadores a escenas de realidad aumentada; basta con disponer de un teléfono móvil o tableta con aplicaciones instaladas del tipo de Layar o Aurasma, entre otras.

\section{B. Recursos}

Para su empleo solo se necesitan ordenadores personales, teléfonos móviles y tabletas con acceso a Internet. El alumno accede a su conveniencia durante su tiempo de estudio al repositorio de problemas disponibles en la web. Pero este sistema también se aplica en el aula durante algunas clases del curso, precisamente cuando se tratan los conceptos básicos que resultan elusivos a la hora de interpretar la respuesta de una estructura ante unas acciones determinadas.

\section{Metodología y técnica}

Aparte de la utilización que hagan los alumnos de este repositorio durante su estudio personal, también en el aula se emplea en sesiones de estudio dirigido, de evaluación por pares y también en proyectos de aula invertida.

En las sesiones de estudio dirigido, se plantea en el aula una clase práctica sobre un problema a resolver individualmente. El profesor indica las líneas generales y fundamentos teóricos de la resolución. Posteriormente los alumnos disponen de un cierto tiempo para resolver individualmente un caso de dicho problema, cada uno con un juego de datos diferente, en función de su número de matrícula, de manera que sea altamente improbable que pueda coincidir un mismo juego de datos en pupitres contiguos. Al finalizar el tiempo establecido, se recogen los ejercicios y se abre un turno de consultas para aclarar dudas sobre la resolución con el profesor; se muestra en el ordenador del aula el ejercicio interactivo disponible en la web, que permite obtener resultados numéricos al instante. Finalmente los alumnos pueden rehacer o completar su ejercicio en caso de ser necesario.

En el proceso de evaluación por pares se repite el sistema anterior hasta que termina el tiempo de resolución; entonces los alumnos entregan su ejercicio y se abre un turno de consultas para aclarar dudas sobre la resolución. Después se vuelven a repartir los ejercicios en orden diferente al de recogida, para llevar a cabo su revisión por pares. Se facilita en ese momento la URL que contiene la versión web del ejercicio interactivo, de forma que los alumnos pueden obtener los resultados numéricos de cualquier juego de datos y así evaluar la realización de su compañero. Estas tareas la pueden hacer desde sus teléfonos móviles o desde el ordenador existente en el aula.

En el proyecto de aula invertida, un grupo de alumnos observa en el laboratorio la realización de una práctica, por ejemplo sobre una prueba de carga de una viga. Posteriormente pueden acceder a la URL en la que se encuentra la página que contiene la simulación numérica de lo que han visto en el laboratorio. Llevan a cabo la experimentación numérica del comportamiento esperado en estructuras análogas a la ensayada y extraen conclusiones y análisis de la respuesta esperada. Posteriormente llevan a cabo una presentación en el aula sobre el estudio de dicha estructura con ayuda de la página web que contiene el simulador.

También está concebido el repositorio web como medio de publicación de los ejercicios resueltos de un examen, de forma que los alumnos puedan disponer de la resolución y del entorno explicativo necesario, nada más terminar el examen.

\section{Ejemplo}

Se muestra a continuación un ejercicio que versa sobre la determinación de las distribuciones de esfuerzos en una viga isostática. Corresponde a la tercera semana del semestre.

\section{Curso: 2016-2017. Práctica 1}

Distribuciones de esfuerzos en vigas simples

En la viga de la figura, existe una articulación en el punto B. Se pide dibujar las leyes de momentos flectores, de esfuerzos cortantes y de esfuerzos axiles.
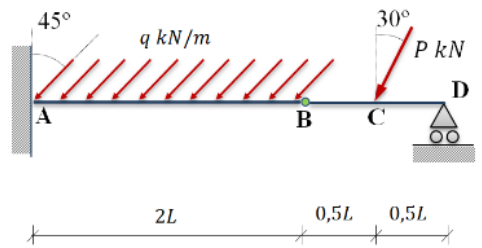

Figura 1: Viga isostática.

Parámetros del problema
\begin{tabular}{|l|l|l|l|l|l|}
\hline Introducir los datos: $q: 30$ & P: 180 & L: 6 & Calcula & Borra \\
\hline
\end{tabular}

Figura 1: Ejercicio de cálculo de leyes de esfuerzos en una viga. Introducción de datos.

En cada problema, el usuario asigna valores a sus datos. Según el caso pueden ser las acciones externas, longitudes de vigas, dimensiones de la sección transversal, etc. En este ejemplo son los valores de las cargas y de longitud de la viga. Al pulsar el botón "Calcula", se obtienen los resultados gráficos y numéricos del problema, como muestra la Figura 2.

Además, para cualquier sección de la viga sobre la que se ubique el puntero del ratón, se indican en la leyenda los valores de los esfuerzos allí actuantes. La simple visualización de las representaciones gráficas suscita al alumno la cuestión sobre las zonas más solicitadas de una estructura para un cierto conjunto de acciones. 


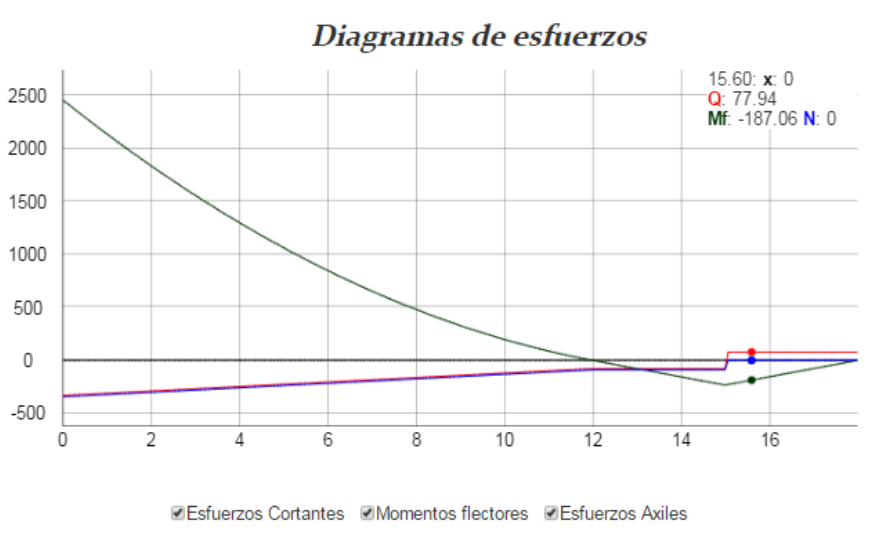

Atrás Cerrar

Figura 2: Representación de las leyes de esfuerzos. La leyenda muestra los valores concretos en cualquier sección.

El usuario puede obtener eventualmente más información acerca de los principios o pasos del proceso de solución pulsando la tecla "h" del teclado, como muestra la Figura 3. El objeto es que cada usuario, a la medida de sus necesidades, pueda cubrir carencias o afianzar sus conocimientos sobre los fundamentos o los pasos que requiere la resolución.

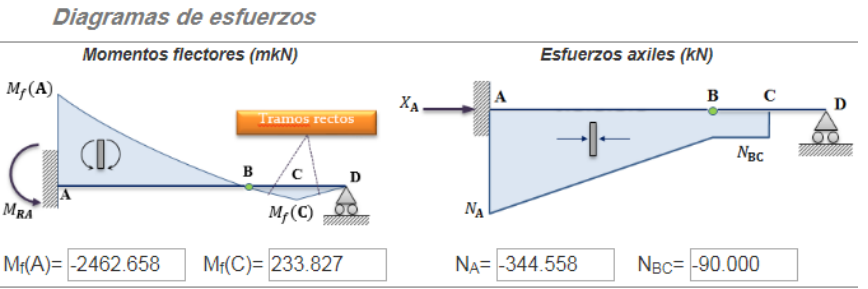

Figura 3: información adicional, disponible bajo demanda.

Mediante vínculos disponibles a lo largo de la página se activan los mini-vídeos explicativos con fundamentos teóricos o aspectos concretos de algún paso de la solución (Figura 4).

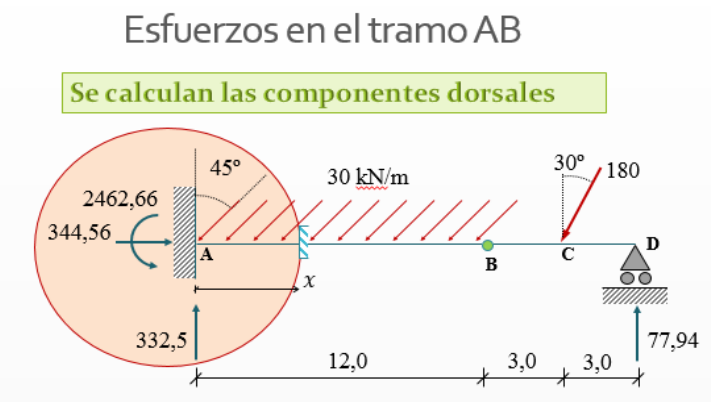

$$
\begin{aligned}
& \text { Figura 2: cálculo de los esfuerzos entre A y B (componente dorsal). } \\
& \left.\begin{array}{c}
N(x)=-X_{A}+30 \frac{\sqrt{2}}{2} x=-344,56+21,213 x \\
Q(x)=-332,5+21,213 x \\
M_{f}(x)=-2462,6+332,5 x-10,607 x^{2}
\end{array}\right\},, 0 \leq x \leq 12
\end{aligned}
$$

Figura 4: El usuario puede obtener adicionalmente consejos o indicaciones para la resolución del problema.
A la vista de las representaciones gráficas, el alumno puede:

- comprobar sus resultados en caso de que haya intentado resolver el problema por su cuenta;

- inferir cuáles son las zonas más solicitadas de la estructura para cada tipo de esfuerzo.

- $\quad$ estimar la respuesta de la estructura ante cada una de las acciones: suprimiendo alguna de las cargas actuantes o bien aplicándolas individualmente, se pueden extraer conclusiones sobre los efectos de cada una en la variabilidad de las leyes de esfuerzos que conlleva.

\section{RESUltadOS}

El impacto que se espera alcanzar es una mejora en el aprendizaje de los alumnos. El sistema lleva un curso en desarrollo. Se ha probado solo para un tema, como medio de aprendizaje alternativo a las tradicionales clases presenciales con un grupo reducido de alumnos. Su destreza en el manejo de la herramienta y el grado de satisfacción mostrado induce a pensar resultados prometedores. Para el próximo curso académico se prevé aplicar para obtener indicadores de logro más objetivos; se empleará un grupo mayor de estudiantes voluntarios y abarcará dos lecciones de la asignatura, de la siguiente forma:

Se imparten en clase durante dos horas los contenidos teóricos correspondientes a una lección. Otro grupo de alumnos estudia dichos contenidos por su cuenta, con el material didáctico disponible así como a través de la web. Los dos grupos de alumnos intentarán resolver a mano en su casa ciertos problemas propuestos de la materia. Se compararán resultados de ambos grupos. Para la segunda lección se invierten los roles de los grupos. Se realizarán además encuestas a los alumnos intervinientes para conocer fortalezas, debilidades y oportunidades de mejora. Una de las repercusiones podrá ser la elaboración de los contenidos teóricos de dichas lecciones en la plataforma web, incluyendo los gadgets multimedia y/o de realidad aumentada para potenciar su eficacia.

Además, a través de encuestas al término del semestre se extrae información sobre la frecuencia, el grado de utilización, de eficacia y de satisfacción por parte de los usuarios. Dicha encuesta versa sobre:

- En qué temas ha sido más útil y beneficiosa la herramienta. Alrededor de dos terceras partes responden que las prácticas de los primeros capítulos son las que más les han servido para adquirir los fundamentos de la asignatura.

- Cuáles son los problemas del repositorio considerados más difíciles. Más de la mitad considera que son los de temas avanzados, correspondientes a la segunda mitad del cuatrimestre.

- Qué necesidades de mejora requiere cada problema del catálogo, a juicio de los usuarios. Alrededor de un 25\% de las ideas o aportaciones se consideran fundamentadas en tanto que mejoran la calidad de los contenidos.

- Qué otras lecciones o conceptos convienen ser implementados en el catálogo web. Las respuestas abarcan desde materias que se consideran preliminares o requisitos previos de la propia asignatura, hasta aspectos 
de asignaturas de cursos posteriores, como es el caso de las líneas de influencia.

- Valoración de la satisfacción del usuario sobre la facilidad y usabilidad de los problemas. En una puntuación de 1 a 5, la media oscila en torno a 3,5.

- Sugerencias o críticas para mejorar la colección. Alrededor de la mitad de los comentarios muestran que carecen de los fundamentos previos para poder aprender eficazmente la asignatura.

\section{CONCLUSIONES}

En este documento se presenta un entorno basado en soporte web, actualmente en desarrollo, implementado sobre código libremente disponible y accesible a través de cualquier navegador de Internet.

La aplicación consiste en un repositorio de problemas interactivos de la asignatura, redactados en español y en inglés de manera que, en cada problema, los estudiantes pueden configurar los datos. El sistema devuelve resultados numéricos y gráficos; así como la posibilidad de acceder, a conveniencia del usuario, a explicaciones ad-hoc intermedias, entre las que se incluyen mini-vídeos ilustrativos. Lo distintivo del método es que facilita que el alumno pueda inferir, evaluar, analizar o estimar aspectos prácticos de la respuesta y del diseño de estructuras sencillas en ingeniería.

Por la forma en que está concebida, la colección de problemas es ampliable y sostenible; para ello solo requiere la codificación en HTML, JavaScript y JQuery, todos ellos estándares libremente disponibles en Internet. Se plantea su aplicabilidad a otras asignaturas tecnológicas básicas de los estudios de ingeniería. Se considera un medio adecuado de cara a su escalabilidad y a su internacionalización como aplicación de las TICs a la enseñanza superior.

El sistema implementado a partir de estas herramientas estándar pretende mejorar el aprendizaje de los alumnos facilitándoles que ejerciten funciones avanzadas de la pirámide, mejorar la calidad de la docencia e introducir en el aula un instrumento para poder realizar evaluaciones por pares, estudio dirigido o prácticas de aula invertida.

\section{AGRADECIMIENTOS}

Los autores desean agradecer el apoyo institucional ofrecido al proyecto IE1617.0406 por parte de la Universidad Politécnica de Madrid en su programa de Ayudas a proyectos de Innovación educativa 2016-17.

\section{REFERENCIAS}

Barrett, P., Davies, F., Zhang, Y. y Barrett, L. (2015). The impact of classroom design on pupils' learning: Final results of a holistic, multi-level analysis. Building and Environment, 89, 118-133. doi:10.1016/j.buildenv .2015.02.013

El-Sawy, K.M.\&Sweedan, A. (2010). Innovative use of computer tools in teaching structural engineering applications. Australasian Journal of Engineering Education 16(1), 35-54

Gallego, M.J., Gámiz, V. y Gutiérrez, E. (2010). El futuro docente ante las competencias en el uso de las tecnologías de la información y comunicación para enseñar. Edutec. Revista electrónica de tecnología educativa, 34, 1-18. doi: http://dx.doi.org/10.21556/ edutec.2010.34.418

Lord T. (2007). Revisiting the Cone of Learning. Is it a Reliable Way to Link Instruction Method with Knowledge Recall? Journal of College Science Teaching, (Nov-Dec), 14-17.

Maquilón, J. J., García-Sánchez, A. y Mirete, A.B. (2014). Webs didácticas en educación superior: análisis de su contenido y valoración del estudiante. Revista Interuniversitaria de Formación del Profesorado, 28(79), 95-114.

Marcé-Nogué, J., Gil, L., Pérez, M. A. y Sánchez, M. (2013). Self-assessment exercises in Continuum Mechanics with autonomous learning. Journal of Technology and Science Education. 3(1), 23-30.

Ministerio de Educación, Cultura y Deporte (2014). Estrategia para la internacionalización de las universidades españolas 2015 - 2020. Recuperado de <http://www.me cd.gob.es/educacion-mecd/dms/mecd/educacion-mecd/ areas-educacion/universidades/politica-internacional/ estrategia-internacionalizacion/ EstrategiaInternacionalizaci-n-Final.pdf >

Mirete, A.B., García, F.A., Sánchez, M.C. (2011). Implicación del alumnado en la valoración de su satisfacción con las web didácticas. Edutec. Revista Electrónica de Tecnología Educativa, 0(37). doi:http://dx.doi.org/ 10.21556/edutec.2011.37.394.

Mosquera-Feijoo, J.C., Plaza-Beltrán, L. \& González-Rodrigo, B. (2015). A framework for adaptive e-learning for continuum mechanics and structural analysis. En IATED (Ed.), INTED2015 9th International Technology, Education and Development Conference (págs. 43764383). Madrid: IATED.

UNESCO. (2015). ICT Competency Framework for Teachers. Recuperado de http://unesdoc.unesco.org/images/0021/ 002134/213475e.pdf 